\title{
Ferramenta interativa para a simulação da propagação de ondas transversais eletromagnéticas em diferentes meios materiais
}

Interactive tool for simulating the propagation of transverse electromagnetic waves in different material media

\author{
Caio Chagas do Amaral ${ }^{1}$, Luciana Antunes Rios ${ }^{* 2}$, Edisio Alves de Aguiar Júnior ${ }^{1,3}$ \\ ${ }^{1}$ Universidade Veiga de Almeida, Rio de Janeiro, RJ, Brasil. \\ ${ }^{2}$ Universidade Federal Fluminense, Escola de Engenharia, Laboratório de Energia e Ambiência, Niterói, RJ, Brasil. \\ ${ }^{3}$ Instituto Nacional de Metrologia, Qualidade e Tecnologia, Diretoria de Metrologia Legal, Duque de Caxias, RJ, Brasil.
}

Recebido em 04 de março de 2021. Revisado em 16 de maio de 2021. Aceito em 15 de junho de 2021.

\begin{abstract}
A experiência tem mostrado que a utilização da tecnologia e de metodologias inovadoras favorece o processo de ensino-aprendizagem. São várias as ferramentas tecnológicas que oferecem a possibilidade de estimular a aprendizagem através da exploração dos conceitos físicos, tais como aplicativos e laboratórios virtuais. Neste trabalho é apresentada uma ferramenta interativa cujo objetivo é auxiliar o processo de ensino-aprendizagem do eletromagnetismo. O aplicativo permite a visualização da propagação de ondas transversais eletromagnéticas em diferentes meios, sendo possível a alteração dos parâmetros que caracterizam a onda e o material. Assim, é possível investigar a propagação de ondas em diferentes cenários e analisar fenômenos tais como o efeito pelicular. Palavras-chave: Eletromagnetismo, Ondas eletromagnéticas, Tecnologias na educação, Simulação no GeoGebra.
\end{abstract}

Experience has shown that the use of technology and innovative methodologies favors the teaching-learning process. There are several technological tools that offer the possibility to stimulate learning through the exploration of physical concepts, such as applications and virtual laboratories. In this work an interactive tool is presented whose objective is to assist the teaching-learning process of electromagnetism. The application allows the visualization of the propagation of transverse electromagnetic waves in different media, making it possible to change the parameters that characterize the wave and the material. Thus, it is possible to investigate the propagation of waves in different scenarios and to analyze phenomena such as the skin effect.

Keywords: Electromagnetism, Electromagnetic waves, Technologies in education, GeoGebra simulation.

\section{Introdução}

O eletromagnetismo é a área da física que estuda, de maneira unificada, os fenômenos elétricos e magnéticos. Sua compreensão permite analisar, em parte, o comportamento da luz e também fenômenos naturais como as auroras boreais, os relâmpagos, e as tempestades magnéticas [1, 2]. Além disso, a teoria eletromagnética está por trás de muitas aplicações médicas e dos processos de geração e distribuição de energia elétrica para as nossas cidades, por exemplo [3]. Por estar associada a tantos aspectos das ciências naturais e tecnológicas, seu estudo é essencial na formação de físicos e engenheiros, entre outros profissionais.

No entanto, a experiência em sala de aula nos mostra que existe uma grande dificuldade, por parte dos alunos, em visualizar e compreender os fenômenos eletromagnéticos. Tal fato é observado já em disciplinas que abordam os fundamentos do eletromagnetismo e se deve,

\footnotetext{
*Endereço de correspondência: lucianarios@id.uff.br
}

entre outras razões, à dificuldade que os alunos possuem em compreender e criar modelos abstratos 4 . Esse é um dos motivos pelos quais o ensino de disciplinas da área de física, como o eletromagnetismo, deve unir teoria e prática experimental [5, 6].

As dificuldades encontradas no aprendizado da teoria eletromagnética também advêm da sua própria natureza, com a qual boa parte dos estudantes não está familiarizada nos anos iniciais da graduação, tanto no que se refere aos fenômenos, como em relação aos conceitos, linguagem, princípios e relações matemáticas [7]. Um exemplo é a dificuldade que os alunos encontram em compreender a natureza da luz: muitos não conseguem interpretá-la como uma onda eletromagnética, o que inclui dificuldades com conceitos (como o comprimento de onda), expressões matemáticas (para os campos elétrico e magnético oscilantes, por exemplo) e representações/modelos (como o diagrama que representa a onda plana eletromagnética) [8-10].

O estudo do eletromagnetismo exige habilidade e conhecimento matemático, bem como a capacidade de 
construir e expressar modelos conceituais utilizando este conhecimento. Portanto, aprender eletromagnetismo significa ser capaz de expressar conceitos físicos através de modelos matemáticos, além de interpretar os modelos já existentes e que formam a base desta área da física. Para tal, é necessário que o aluno tenha um bom conhecimento da matemática envolvida e dos conceitos físicos, mas também seja capaz de desenvolver um certo grau de abstração e visão espacial. Nesse aspecto, o uso de tecnologias e metodologias ativas tem sido de vital importância [11-13].

É cada vez mais evidente que a utilização de ferramentas e metodologias inovadoras, aliadas ao bom desenvolvimento dos conceitos teóricos, favorece o processo de ensino-aprendizagem 12 14. Em conjunto com a tecnologia, são várias as ferramentas que oferecem a possibilidade de estimular a aprendizagem através da exploração dos conceitos físicos, tais como aplicativos, laboratórios virtuais, simulações e games, entre outros 11, 14 18. A utilização de ferramentas interativas transforma o aluno em um sujeito ativo no processo de ensino-aprendizagem, e não um mero receptor do conhecimento a ser transmitido pelo professor.

Seja qual for a abordagem utilizada em sala de aula, a ideia é tentar melhorar o processo de ensinoaprendizagem. Tanto as iniciativas inovadoras baseadas na tecnologia, como as pedagógicas (ou as que misturam ambos os princípios), têm como objetivo comum possibilitar aos estudantes a visualização e a expressão concreta de conceitos abstratos do eletromagnetismo e outras áreas da física [18. No caso específico de animações e simulações, estas podem ser extremamente úteis na representação de fenômenos dinâmicos, como o movimento de um projétil, por exemplo. As simulações podem representar ou modelar objetos reais ou imaginários, sistemas ou fenômenos, e possuem alguns benefícios, como fornecer feedback rápido para aperfeiçoar a compreensão de certos conceitos e permitir aos estudantes gerarem e testarem hipóteses. Elas também podem ser bastante úteis quando um experimento não puder ser reproduzido pelos estudantes, seja no caso de situações perigosas ou realizações muito caras. No entanto, existe uma diferença significativa entre experimentar um fenômeno, através de um experimento real, e uma simulação computacional. A não percepção de tal diferença pode acarretar concepções equivocadas sobre o fenômeno em questão. Além disso, toda simulação é baseada em um modelo, que pode conter erros ou até não ser simulado corretamente [11. Portanto, ao utilizarmos uma simulação, devemos ter em mente que esta é uma ferramenta auxiliar no processo de ensino-aprendizagem, e que a mesma deve ser construída com cuidado e para cumprir certos objetivos. Deve ficar claro que seu papel não é substituir todo um conjunto de outras abordagens que podem ser utilizadas com o mesmo tema.

O objetivo deste trabalho é apresentar uma ferramenta interativa que permite visualizar a propagação de ondas eletromagnéticas em diferentes meios materiais. $\mathrm{O}$ estudo das ondas eletromagnéticas é importante para a compreensão de diversos fenômenos físicos, além de formar a base teórica de áreas aplicadas, como a engenharia de telecomunicações. Pela importância do assunto, e também devido ao alto grau de dificuldade encontrado pelos alunos em compreender este fenômeno, o tópico foi escolhido como o primeiro a ser abordado dentro de um projeto mais amplo, que terá como objetivo desenvolver ferramentas interativas para auxiliar no estudo do eletromagnetismo. Até onde foi possível pesquisar, não foram obtidas quaisquer informações sobre ferramentas interativas que funcionem com os mesmos parâmetros definidos para este trabalho.

O uso de aplicativos e ferramentas interativas, que permitem a inserção de dados pelo usuário e a visualização dinâmica dos fenômenos físicos, pode ser um forte aliado no processo de ensino-aprendizagem. A ferramenta apresentada neste trabalho foi desenvolvida no GeoGebra, um aplicativo de matemática dinâmica disponível em várias plataformas. As razões para a escolha do GeoGebra, assim como uma breve discussão sobre o desenvolvimento da ferramenta são apresentadas na seção 3, após uma pequena revisão sobre a propagação de ondas transversais eletromagnéticas em meios materiais (seção 2). Na seção 4 serão apresentados alguns resultados obtidos com a ferramenta e, em seguida, algumas conclusões e perspectivas (seção 5 ).

\section{Ondas Transversais Eletromagnéticas em Meios Materiais}

O modo transversal eletromagnético (TEM) é caracterizado por ondas transversais eletromagnéticas, isto é, ondas cuja direção de propagação é perpendicular ao plano de oscilação dos campos elétrico e magnético, que também são perpendiculares entre si. O estudo do modo TEM é o primeiro passo em uma investigação mais profunda sobre a propagação de ondas eletromagnéticas em meios materiais pelo fato das ondas no espaço livre (ou em dielétricos isotrópicos) poderem ser representadas pela superposição de ondas planas. Além disso, o modo TEM também ocorre em ondas confinadas em guias de onda e fibras óticas, o que torna o seu estudo importante para a área de telecomunicações, por exemplo [3, 19].

Uma onda TEM linearmente polarizada que se propaga em um dielétrico imperfeito ao longo de $+\widehat{\boldsymbol{a}}_{\boldsymbol{x}}$ e cujo campo elétrico oscila ao longo de $z$ pode ser representada pela função de onda [3, 19, 20,

$$
\vec{E}(x, t)=E_{0} e^{-\alpha x} \cos (\omega t-\beta x) \widehat{\boldsymbol{a}}_{\boldsymbol{z}}
$$

onde $E_{0}$ é a amplitude do campo elétrico (em $\mathrm{V} / \mathrm{m}$ no Sistema Internacional, SI), $\omega$ é a frequência angular da onda $(\mathrm{em} \mathrm{rad} / \mathrm{s}), \beta$ é o número de onda $(\mathrm{em} \mathrm{rad} / \mathrm{m})$ e $\alpha$ é a constante de atenuação (em Nepers por metro, $\mathrm{Np} / \mathrm{m})$. À medida que a onda se propaga em um 
dielétrico imperfeito (com perdas), ela transfere parte da sua energia para o meio devido à condutividade $(\sigma)$ do mesmo. Assim, $\alpha$ é uma medida da taxa de decaimento espacial da onda: conforme ela se propaga ao longo de $x$, sua amplitude é atenuada por um fator $e^{-\alpha x}$. Os dielétricos imperfeitos possuem condutividade baixa, mas não nula. A partir da análise da propagação de ondas nesse tipo de material (caso geral) é possível derivar os casos especiais de propagação em dielétricos perfeitos $(\sigma=0)$ e condutores $(\sigma \cong \infty)$.

Uma vez que os campos elétrico e magnético da onda devem estar contidos no mesmo plano, o qual é perpendicular à direção de propagação, o produto vetorial dos versores que indicam as direções de oscilação dos campos deve obedecer à regra $\widehat{\boldsymbol{a}}_{\boldsymbol{E}} \times \widehat{\boldsymbol{a}}_{\boldsymbol{H}}=\widehat{\boldsymbol{a}}_{\boldsymbol{k}}$, onde $\widehat{\boldsymbol{a}}_{\boldsymbol{k}}$ é o versor que indica a direção de propagação da onda. Para a onda em questão, $\widehat{\boldsymbol{a}}_{\boldsymbol{E}}=\widehat{\boldsymbol{a}}_{\boldsymbol{z}}$ e $\widehat{\boldsymbol{a}}_{\boldsymbol{k}}=\widehat{\boldsymbol{a}}_{\boldsymbol{x}}$, $\operatorname{logo} \widehat{\boldsymbol{a}}_{\boldsymbol{H}}=-\widehat{\boldsymbol{a}}_{\boldsymbol{y}}$. Assim, a função que representa o campo magnético da onda é

$$
\vec{H}(x, t)=-H_{0} e^{-\alpha x} \cos \left(\omega t-\beta x-\theta_{\eta}\right) \widehat{\boldsymbol{a}}_{\boldsymbol{y}}
$$

onde $H_{0}$ é a amplitude do campo magnético (em $\mathrm{A} / \mathrm{m}$ ) e $\theta_{\eta}$ é o ângulo de fase associado à impedância do meio (em radianos). As amplitudes dos campos elétrico e magnético estão relacionadas através da expressão

$$
H_{0}=\frac{E_{0}}{|\eta|}
$$

onde $\eta$ é a impedância intrínseca do meio (em ohms, $\Omega$ ). Por ser uma quantidade complexa, pode ser escrita na forma

$$
\eta=|\eta| e^{j \theta_{\eta}}
$$

e seu módulo é dado pela expressão

$$
|\eta|=\frac{\sqrt{\frac{\mu}{\varepsilon}}}{\left[1+\left(\frac{\sigma}{\omega \varepsilon}\right)^{2}\right]^{\frac{1}{4}}}
$$

onde $\varepsilon$ e $\mu$ são, respectivamente, a permissividade elétrica (em Farad por metro, F/m) e a permeabilidade magnética (em Henry por metro, $\mathrm{H} / \mathrm{m}$ ) do meio, e a condutividade $\sigma$ é dada em Siemens por metro $(\mathrm{S} / \mathrm{m})$. O ângulo de fase $\theta_{\eta}$ representa a defasagem entre os campos elétrico e magnético da onda, e obedece à relação

$$
\tan 2 \theta_{\eta}=\frac{\sigma}{\omega \varepsilon}
$$

Além disso, para uma onda TEM que se propaga em um meio qualquer,

$$
0 \leq \theta_{\eta} \leq 45^{\circ}
$$

Observando as equações (1) e (2), verificamos que $\vec{H}$ se atrasa em relação a $\vec{E}$ (ou $\vec{E}$ se adianta em relação a $\vec{H})$. Para os dielétricos perfeitos (sem perdas), $\sigma=0 \mathrm{e}$ $\theta_{\eta}=0$, de acordo com a equação (6). Já para materiais que possuem condutividades maiores, a transferência de energia da onda para o meio tende a crescer, dependendo da frequência da onda. Nesses casos, a defasagem entre os campos elétrico e magnético é não nula e pode se aproximar de $45^{\circ}$. Se o meio onde a onda se propaga for um condutor $(\sigma \rightarrow \infty)$, é possível verificar pela equação (6) que $\tan 2 \theta_{\eta} \rightarrow \infty$ e, portanto, $\theta_{\eta} \approx 45^{\circ}$.

Ainda sobre a equação (6), a quantidade $\sigma / \omega \varepsilon$, chamada de tangente de perdas (adimensional), pode ser usada para quantificar as perdas em um meio. Assim, um meio material pode ser considerado um dielétrico perfeito se $\sigma / \omega \varepsilon \ll 1$, ou um bom condutor se $\sigma / \omega \varepsilon \gg 1$. Portanto, no que se refere à propagação de ondas, o comportamento característico de um meio não depende apenas dos seus parâmetros constitutivos $\varepsilon, \mu$ e $\sigma$, mas também da frequência $f$ da onda, a qual está relacionada à fonte.

A tangente de perdas também é utilizada no cálculo das grandezas $\alpha$ e $\beta$,

$$
\begin{aligned}
& \alpha=\omega \sqrt{\frac{\mu \varepsilon}{2}\left[\sqrt{1+\left(\frac{\sigma}{\omega \varepsilon}\right)^{2}}-1\right]} \\
& \beta=\omega \sqrt{\frac{\mu \varepsilon}{2}\left[\sqrt{1+\left(\frac{\sigma}{\omega \varepsilon}\right)^{2}}+1\right]}
\end{aligned}
$$

$\beta$ é uma medida do deslocamento de fase por unidade de comprimento, enquanto a frequência angular $\omega$ representa o deslocamento de fase por unidade de tempo. A fase da onda representada pela equação (1), por exemplo, é a quantidade $(\omega t-\beta x)$. Note que, para $\sigma / \omega \varepsilon=0$ (dielétricos perfeitos), $\alpha=0$ e a onda não sofre atenuação.

\section{Desenvolvimento da Ferramenta}

A ferramenta desenvolvida simula a propagação do modo TEM em um determinado meio a partir de parâmetros de entrada associados à onda e ao meio. A escolha do GeoGebra (https://www.geogebra.org/) para o desenvolvimento da ferramenta se deve à gratuidade da sua licença e também ao fato do aplicativo estar disponível para várias plataformas, podendo ser instalado em computadores, tablets e celulares. O aplicativo também pode ser utilizado diretamente no navegador, sem a necessidade de instalação. Os materiais didáticos desenvolvidos com o GeoGebra ficam armazenados no próprio aplicativo, onde é possível encontrar diversas ferramentas interativas desenvolvidas para auxiliar no processo de ensino-aprendizagem em áreas como cálculo, física e estatística.

O fato de possuir uma licença GPL (General Public License), isto é, uma licença livre, permite que os materiais desenvolvidos com o GeoGebra sejam utilizados e distribuídos livremente, tornando-os alinhados com as 

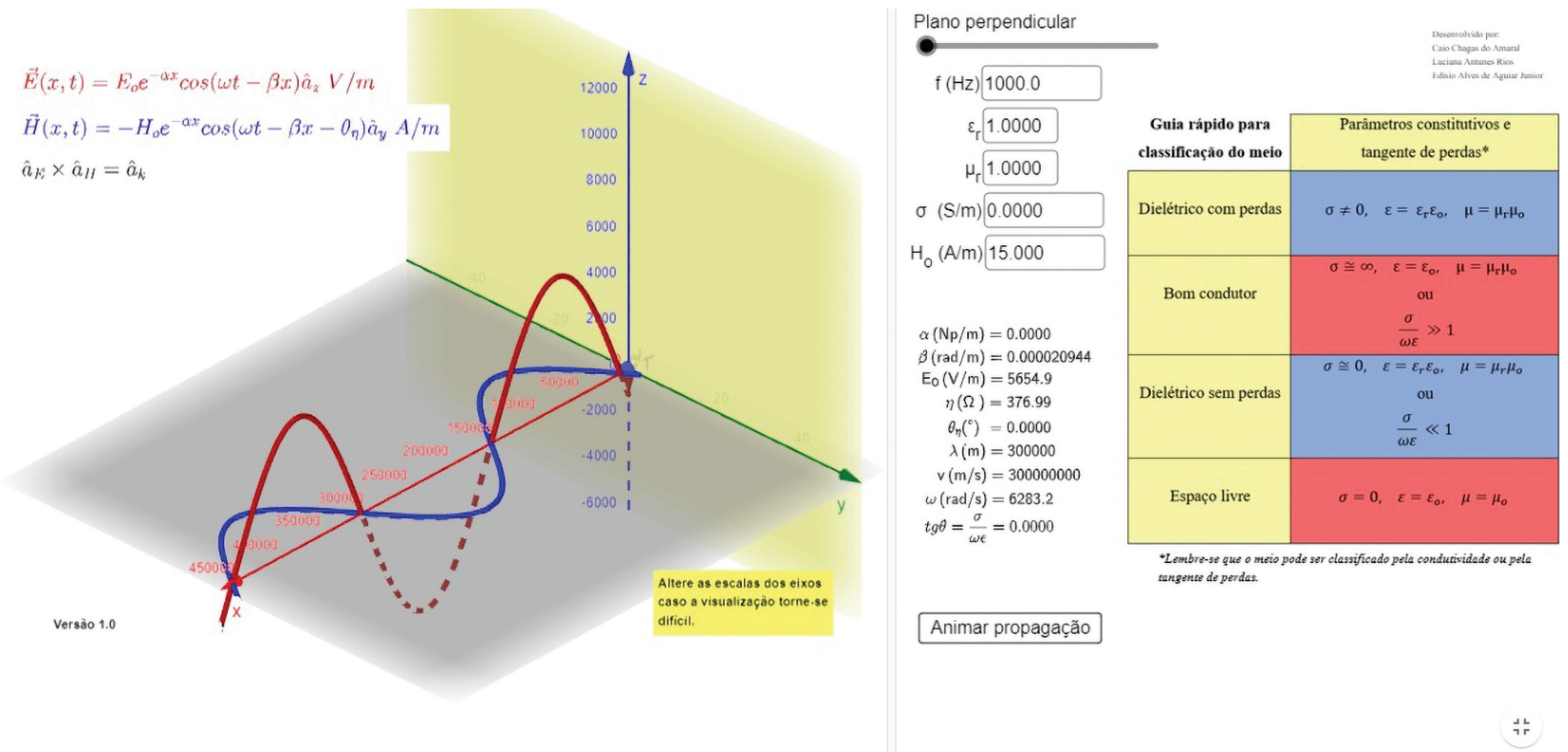

Figura 1: Interface do aplicativo no GeoGebra.

perspectivas da educação aberta [21], movimento que trabalha pelo livre acesso a oportunidades de aprendizagem. Outros exemplos de como o GeoGebra pode ser explorado no ensino de física podem ser encontrados no site https://ophysics.com/, criado e mantido pelo Professor Tom Walsh, e na referência 22.

Os parâmetros de entrada associados à onda são a frequência $f$, em hertz $(\mathrm{Hz})$, e a amplitude do campo magnético, $H_{0}$. Já os parâmetros que caracterizam o meio são, além da condutividade $\sigma$, a permissividade e permeabilidade relativas, definidas respectivamente como $\varepsilon_{r}=\varepsilon / \varepsilon_{0}$ e $\mu_{r}=\mu / \mu_{0}$ (lembrando que $\varepsilon_{0}$ e $\mu_{0}$ são a permissividade e permeabilidade do espaço livre). Todos esses parâmetros são fornecidos pelo usuário, que então pode simular a propagação de ondas de diferentes frequências em diferentes meios materiais.

A Figura 1 mostra a tela que será visualizada no instante em que o aplicativo for aberto. No lado direito é possível visualizar os 5 parâmetros de entrada, além dos parâmetros $\alpha, \beta,|\eta|, \theta_{\eta}$ e $E_{0}$, todos determinados a partir dos dados fornecidos utilizando as equações apresentadas na seção 2 (observe que, nesta versão do aplicativo, as direções de propagação e dos campos $\vec{E}$ e $\vec{H}$ são mantidas fixas). Além destes, também são calculados o comprimento de onda $\lambda$ (em metros), a velocidade de fase $v(\mathrm{em} \mathrm{m} / \mathrm{s})$ e $\omega$, a partir das relações

$$
\begin{aligned}
\lambda & =\frac{2 \pi}{\beta} \\
v & =\frac{\omega}{\beta} \\
\omega & =2 \pi f
\end{aligned}
$$

A tangente de perdas também é apresentada na forma

$$
\tan \theta=\frac{\sigma}{\omega \varepsilon}
$$

onde $\theta=2 \theta_{\eta}$ é o ângulo de perdas. Os campos com os parâmetros de entrada e as grandezas calculadas são mostrados em destaque na Figura 2, onde também é possível visualizar um botão que permite animar a propagação da onda, apresentada no lado esquerdo da tela na Figura 1

Também na Figura 2 é apresentado um controle deslizante que permite mover o plano perpendicular que contém os vetores campo elétrico e campo magnético da onda, mostrada em destaque na Figura 3. A onda é construída a partir dos dados de entrada e das grandezas calculadas, e apesar das direções de propagação e de oscilação dos campos terem sido mantidas fixas para este trabalho (equações (1) e (2)), é possível girar o sistema de eixos e mudar a perspectiva de observação na janela tridimensional.

No lado direito da Figura 1 também é possível visualizar uma tabela para auxiliar na classificação do material de acordo com os parâmetros constitutivos do meio ou com a tangente de perdas (Tabela 1). Lembre-se que um material que, em princípio, é um dielétrico imperfeito (condutividade finita, mas baixa) pode se comportar como um bom condutor dependendo da frequência da onda, por exemplo.

Junto com o aplicativo são disponibilizados dois materiais auxiliares: um vídeo para auxiliar na manipulação da ferramenta, e um texto com uma pequena revisão sobre a propagação de ondas TEM em meios materiais e também algumas dicas para a utilização do aplicativo. O principal objetivo do vídeo é ajudar o usuário na 


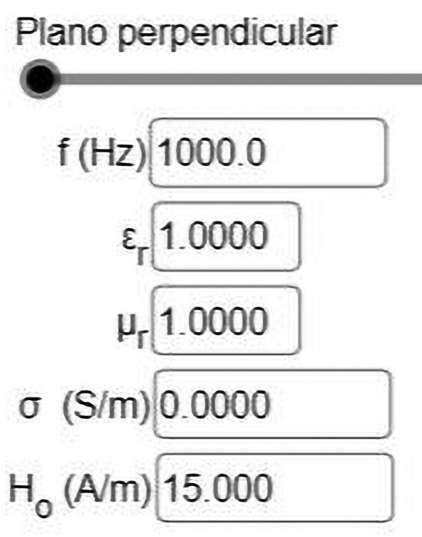

$$
\begin{aligned}
\alpha(\mathrm{Np} / \mathrm{m}) & =0.0000 \\
\theta(\mathrm{rad} / \mathrm{m}) & =0.000020944 \\
\mathrm{E}_{0}(\mathrm{~V} / \mathrm{m}) & =5654.9 \\
\eta(\Omega) & =376.99 \\
\theta_{\pi}\left(^{\circ}\right) & =0.0000 \\
\lambda(\mathrm{m}) & =300000 \\
v(\mathrm{~m} / \mathrm{s}) & =300000000 \\
\omega(\mathrm{rad} / \mathrm{s}) & =6283.2 \\
\operatorname{tg} \theta=\frac{\sigma}{\omega \varepsilon} & =0.0000
\end{aligned}
$$

\section{Animar propagação}

Figura 2: Parâmetros de entrada e saída do aplicativo e controles para o deslocamento do plano perpendicular e animação da onda.

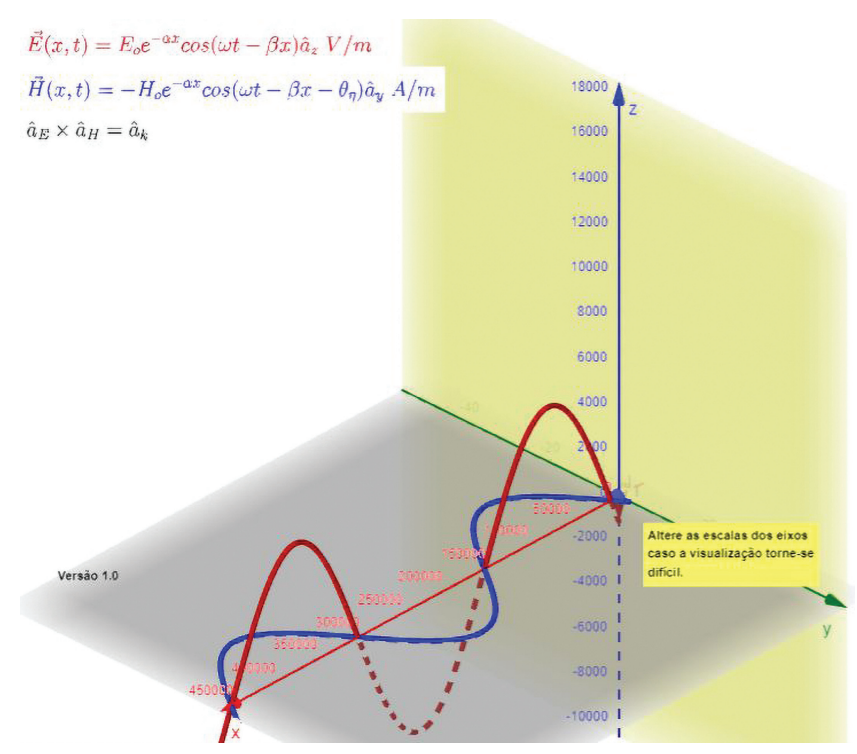

Figura 3: Janela mostrando a propagação da onda eletromagnética ao longo do eixo $x$, com o campo elétrico oscilando ao longo de $z$ e o campo magnético ao longo de $y$.
Tabela 1: Guia rápido para a classificação do meio de propagação [19].

\begin{tabular}{lc}
$\begin{array}{l}\text { Guia rápido para } \\
\text { classificação do meio }\end{array}$ & $\begin{array}{c}\text { Parâmetros constitutivos e } \\
\text { tangente de perdas }\end{array}$ \\
\hline $\begin{array}{l}\text { Dielétrico com perdas } \\
\text { Bom condutor }\end{array}$ & $\begin{array}{c}\sigma \neq 0, \varepsilon=\varepsilon_{r} \varepsilon_{0}, \mu=\mu_{r} \mu_{0} \\
\sigma \cong \infty, \varepsilon=\varepsilon_{0}, \mu=\mu_{r} \mu_{0} \\
\text { ou }\end{array}$ \\
& $\begin{array}{c}\sigma / \omega \varepsilon \gg 1 \\
\text { Dielétrico sem perdas }\end{array}$ \\
& $\begin{array}{c}\sigma \cong 0, \varepsilon=\varepsilon_{r} \varepsilon_{0}, \mu=\mu_{r} \mu_{0} \\
\text { ou } \\
\text { Espaço livre }\end{array}$ \\
\hline
\end{tabular}

alteração das escalas dos eixos caso a visualização da onda se torne difícil devido a mudanças nos parâmetros de entrada e, consequentemente, nas características da onda e do meio. Para acessar o aplicativo basta clicar no link https://www.geogebra.org/m/pwux2qkn

É importante ressaltar que há uma limitação no aplicativo: ele só funciona bem até frequências de, aproximadamente, $1 \mathrm{GHz}$. A partir deste valor de $f$ as operações (cálculos e animação da onda) parecem ficar muito pesadas para o GeoGebra. No entanto, esta limitação não impede que a ferramenta seja utilizada para auxiliar no processo de ensino-aprendizagem e na análise de alguns efeitos associados à propagação de ondas, como veremos a seguir.

\section{Resultados}

O objetivo do aplicativo é auxiliar alunos e professores no processo de ensino-aprendizagem do eletromagnetismo. Nesta seção são discutidos alguns efeitos/fenômenos relacionados à propagação de ondas eletromagnéticas que podem ser observados com a utilização da ferramenta. Espera-se que, ao visualizar e interagir com conceitos físicos discutidos em sala e nos livros didáticos, o aluno amplie sua compreensão sobre os fenômenos em questão e sua percepção sobre o eletromagnetismo em geral.

A ferramenta permite ao usuário visualizar a propagação de ondas TEM de diferentes frequências, em diferentes meios. Fixando o valor da frequência, por exemplo, é possível verificar como o comprimento de onda, a velocidade e a relação entre as amplitudes dos campos elétrico e magnético são afetados pelas mudanças no meio. Isto pode ser observado nas Figuras 4 e 5 Na Figura 4 observamos uma onda com $f=1$ $\mathrm{kHz}$ e $H_{0}=100 \mathrm{~mA} / \mathrm{m}$ se propagando no espaço livre $\left(\sigma=0, \varepsilon_{r}=1, \mu_{r}=1\right)$. Já na Figura 5 observamos a mesma onda se propagando em um dielétrico sem perdas $\left(\sigma \cong 0, \varepsilon_{r}=81, \mu_{r} \cong 1\right)$. Note como $\lambda, v$ e a relação entre $E_{0}$ e $H_{0}$ são alterados devido à mudança de meio (a escala foi alterada na Figura 5 para melhor visualização). Em ambos os casos a atenuação da onda é desprezível, pois $\sigma / \omega \varepsilon \ll 1$.

Ainda sobre os casos mostrados nas Figuras 4 e 5 vale ressaltar a variação da velocidade da onda com o 

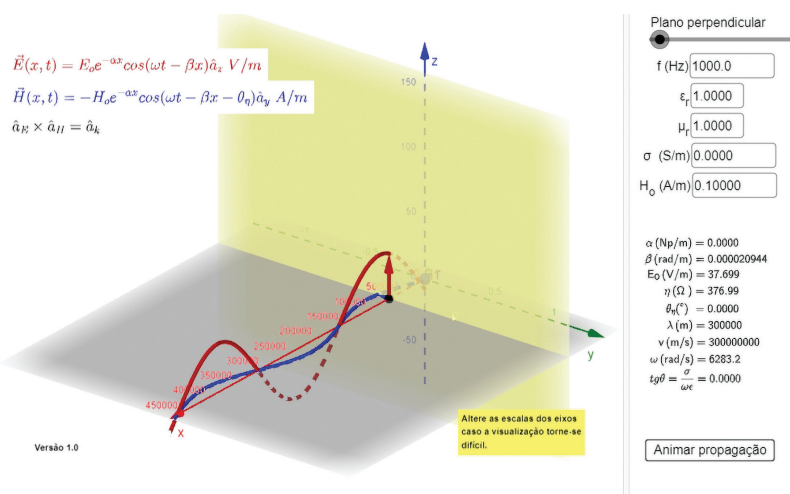

Figura 4: Onda TEM se propagando no espaço livre.

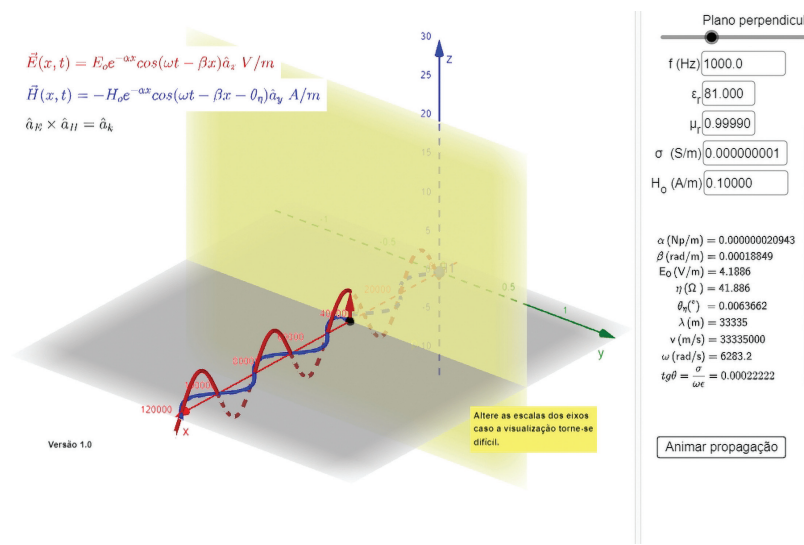

Figura 5: Onda TEM se propagando em um dielétrico sem perdas.

meio. Alguns trabalhos indicam que estudantes nos anos iniciais da graduação acreditam não existir dependência da velocidade da luz com o meio [10]. A ferramenta aqui apresentada pode ajudar no esclarecimento de dúvidas como essa, uma vez que permite ao aluno analisar a propagação em diferentes cenários, possibilitando assim a avaliação do impacto do meio sobre determinadas grandezas associadas à onda. Uma outra questão que pode ser trabalhada com a utilização da ferramenta é a representação da onda eletromagnética. Estudos indicam que os estudantes possuem grande dificuldade em interpretar e reproduzir os diagramas representativos das ondas TEM, como os mostrados nas Figuras 4 e 5 [ 9 . Isso dificultaria, inclusive, a compreensão, por parte dos alunos, de que a luz é uma onda eletromagnética. Entre as dificuldades apontadas podemos mencionar a ideia de que os campos elétrico e magnético estão confinados às regiões limitadas pelas curvas senoidais que os representam, e a não compreensão da interdependência entre $\vec{E}$ e $\vec{H}$ e do fato de que uma onda eletromagnética não pode consistir em apenas um deles.

As Figuras 6 e 7 mostram ondas de frequências diferentes se propagando em um mesmo material e a mudança de comportamento do meio de acordo com

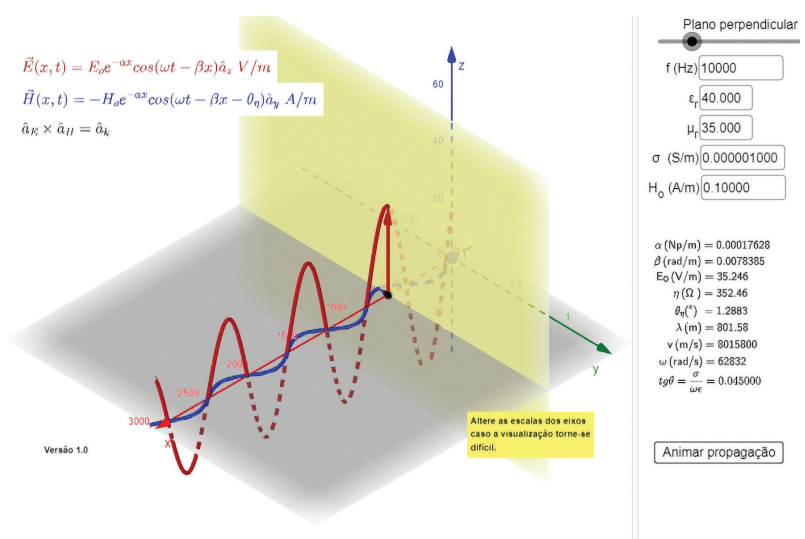

Figura 6: Onda TEM se propagando em um dielétrico atenuação leve.

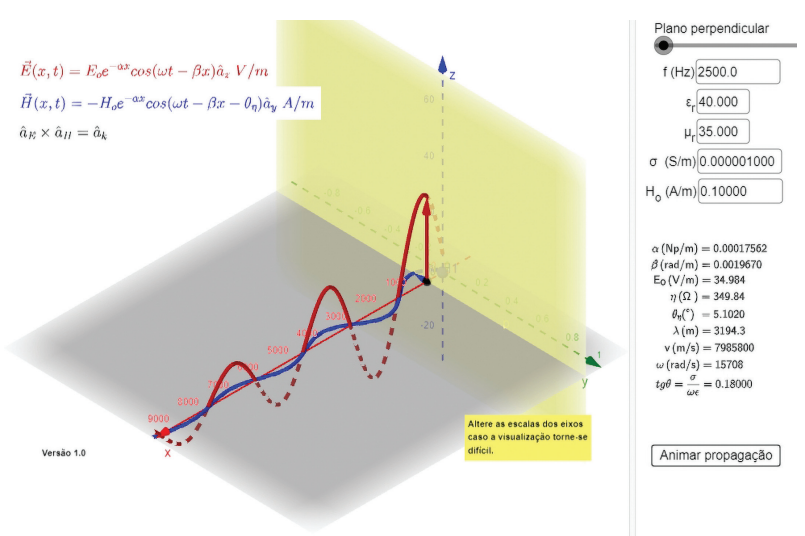

Figura 7: Onda TEM se propagando em um dielétrico com perdas.

a frequência. A Figura 6 mostra uma onda com $f=$ $10 \mathrm{kHz}$ e $H_{0}=100 \mathrm{~mA} / \mathrm{m}$ se propagando em um dielétrico $\operatorname{com} \varepsilon_{r}=40, \mu_{r}=35$ e condutividade muito baixa, $\sigma=1 \mu \mathrm{S} / \mathrm{m}$. Observe que, nesse caso, a tangente de perdas não é tão desprezível $(\sigma / \omega \varepsilon=0,045)$ e a onda sofre uma leve atenuação. Já na Figura 7 a situação é outra: uma onda com $f=2,5 \mathrm{kHz}$ se propaga no mesmo meio que a anterior, mas sofre uma atenuação mais intensa. Isso se deve ao aumento da tangente de perdas $(\sigma / \omega \varepsilon=0,18)$ acarretado pela diminuição da frequência. Portanto, um mesmo material pode apresentar comportamentos distintos, dependendo da frequência da onda que nele se propaga. Observe também a mudança no valor do ângulo de fase em relação às Figuras $4 \mathrm{e} 5$ onde temos $\theta_{\eta} \approx 0$ : devido ao aumento da tangente de perdas nas situações representadas nas Figuras 6 e 7, a defasagem entre os campos também cresce, o que é evidenciado pelo aumento de $\theta_{\eta}$. Aqui as escalas também foram alteradas para melhor visualização.

Por fim, as Figuras 8 e 9 mostram ondas TEM de mesma frequência e amplitude $H_{0}$ se propagando em dois materiais com condutividades finitas, mas diferentes. 


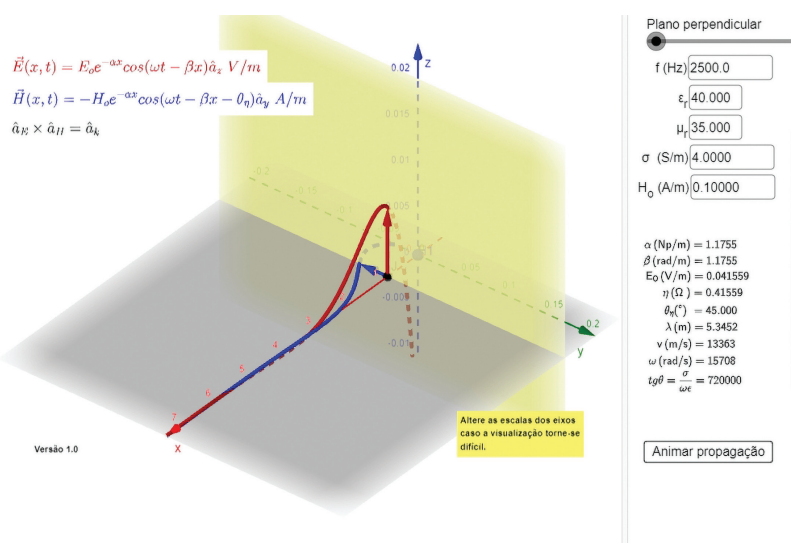

Figura 8: Onda TEM com forte atenuação em um material com $\sigma=4 \mathrm{~S} / \mathrm{m}$.

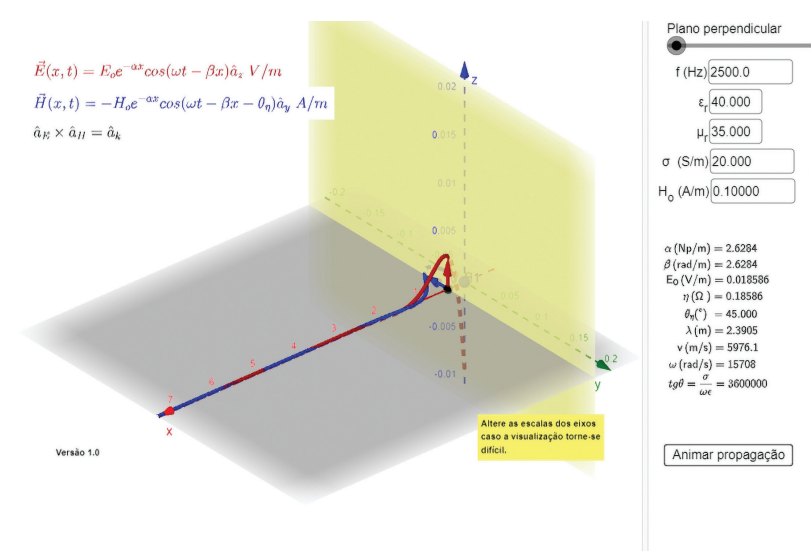

Figura 9: Onda TEM com forte atenuação em um material com $\sigma=20 \mathrm{~S} / \mathrm{m}$.

Note que, na Figura 8, a onda sofre uma forte atenuação, não sendo capaz de completar 1 período. Neste caso, o material tem condutividade baixa, $\sigma=4 \mathrm{~S} / \mathrm{m}$, mas devido ao alto valor da tangente de perdas (acarretado pelo valor de $f$ ), o meio se comporta como um bom condutor. Na Figura 9 a atenuação é ainda mais intensa, o que é esperado devido ao valor mais alto da condutividade $(\sigma=20 \mathrm{~S} / \mathrm{m})$ e $\sigma / \omega \varepsilon \rightarrow \infty$ (escalas foram alteradas para melhorar visualização). Devido à forte atenuação que as ondas TEM sofrem em bons condutores, dificilmente elas se propagam neste tipo de material. Este fenômeno, conhecido como efeito pelicular, também afeta correntes alternadas e é o responsável pelo aumento da resistência aparente de um condutor elétrico a esse tipo de corrente. Observe o valor de $\theta_{\eta}$ nas Figuras 8 e 9 próximo de $45^{\circ}$, o que é esperado para bons condutores.

\section{Conclusões e Perspectivas}

Neste trabalho foi apresentada uma ferramenta interativa cujo objetivo é auxiliar no processo de ensino- aprendizagem do eletromagnetismo, mais precisamente na compreensão da propagação de ondas TEM em diferentes tipos de materiais. A ferramenta foi desenvolvida no GeoGebra com o intuito de facilitar o acesso de professores e estudantes a mesma, visto que o aplicativo de matemática dinâmica se encontra disponível para várias plataformas e pode ser acessado através de computadores e celulares. Também foram apresentados alguns resultados obtidos com a ferramenta, mostrando assim o seu potencial para auxiliar os alunos na resolução de exercícios e na compreensão dos fenômenos estudados.

O próximo passo é tentar avaliar o impacto da ferramenta no processo de aprendizagem dos alunos. Para tal, serão aplicados questionários (pré-teste e pósteste) a algumas turmas do curso de Engenharia Elétrica da Universidade Veiga de Almeida. Espera-se que a partir da análise estatística das respostas seja possível avaliar a influência da ferramenta na compreensão dos alunos sobre a propagação de ondas e fenômenos associados. Além disso, os resultados do levantamento podem nos ajudar a melhorar a própria ferramenta e a traçar estratégias para o desenvolvimento de outros aplicativos.

\section{REFERÊNCIAS}

[1] J.D. Jackson, Classical Electrodynamics (John Wiley \& Sons, New York, 1998).

[2] D.A. Gurnett e A. Bathacharjee, Introduction to Plasma Physics (Cambridge University Press, Cambridge, 2017).

[3] W.H. Hayt, Jr e J.A. Buck, Engineering Electromagnetics (McGraw-Hill, New York, 2005).

[4] C. Tres e S.A. dos Santos, Os Desafios da Escola Pública Paranaense na Perspectiva do Professor PDE (Cadernos PDE, Irati, 2016).

[5] J.M. Cardoso e M. Zannin, Rev. Bras. Ensino Fís. 41, e20190028 (2019).

[6] T.R. Barros e W.S. Dias, Rev. Bras. Ensino Fís. 41, e20190049 (2019).

[7] D.P. Maloney, T.L. O'Kuma, C.J. Hieggelke e A. Van Heuvelen, Phys. Educ. Res., Am. J. Phys. Suppl. 69, S12 (2001).

[8] P. Colin e L. Viennot, Phys. Educ. Res., Am. J. Phys. Suppl. 69, S36 (2001).

[9] B.S. Ambrose, P.R.L. Heron, S. Vokos e L.C. McDermott, Am. J. Phys. 67, 891 (1999).

[10] B. Djanette e C. Fouad, Procedia - Soc. Behav. Sci. 152, $582(2014)$.

[11] A. Medeiros e C.F. de Medeiros, Rev. Bras. Ensino Fís. 24, 77 (2002).

[12] V. Heckler, M.F.O. Saraiva e K.S. Oliveira Filho, Rev. Bras. Ensino Fís. 29, 267 (2007).

[13] M.P. Quibao, A.C. Silva, N.S. de Almeida, R.M.A.A. Silva, S.R. Muniz e F.F. Paiva, Rev. Bras. Ensino Fís. 41, e20180258 (2019).

[14] J.B. Silva, G.L. Sales e J.B. Castro, Rev. Bras. Ensino Fís. 41, e20180309 (2019).

[15] J.M.M. Oliveira, M. Ferreira e D. Mill, Inc. Soc. 10, 147 (2016). 
[16] L. de la Torre, J. Sánchez, S. Dormido, J.P. Sánchez, M. Yuste e C. Carreras, Eur. J. Phys. 32, 571 (2011).

[17] A. Bjelopera, M. Kajinié e M. Dumié, Int. J. Educ. Inf. Technol. 13, 73 (2019).

[18] H. Roussel e M. Hélier, Adv. Electromagn. 1, 65 (2012).

[19] M.N.O. Sadiku, Elements of Electromagnetics (Oxford University Press, Oxford, 2014).

[20] L.A. Rios, Campos e Ondas (e-book) (UVA - Ilumno, Rio de Janeiro, 2018).

[21] D. Furtado e T. Amiel, Guia de Bolso da Educação Aberta (CEAD - UnB, Brasília, 2020).

[22] L. Solvang e J. Haglund, em: Proceedings of 10th International Conference on Education and New Learning Technologies (Palma, 2018). 